\title{
REINVENCIÓN EN LA EXPERIENCIA EDUCATIVA PRESENCIAL Y VIRTUAL
}

\section{REINVENTION IN THE FACE-TO-FACE AND VIRTUAL EDUCATIONAL EXPERIENCE}

\author{
Oswaldo Medaglia Zapata \\ Semillero de investigación SIMEC \\ Institución Universitaria Antonio José Camacho \\ Recibido: 03/05/2020 Aprobado: 25/05/2021 \\ Cómo citar este artículo: \\ Medaglia Zapata, O.(2021). Reinvención en la experiencia educativa presencial y virtual. Revista Sapientía, $13(25)$, 16 - 30.
}

\section{RESUMEN}

Con esta investigación se pretende demostrar la experiencia significativa en la labor docente universitaria en modalidad presencial con apoyo en la aplicación de Google Classroom ${ }^{\circledast}\left(\mathrm{GC}^{\circledast}\right)$. Se considera caso de estudio para ser incluido como metodología alternativa de aprendizaje y complementaria en la modalidad presencial y en la virtualización en las Instituciones de Educación Superior (IES). La metodología desarrollada consistió en el diseño detallado de un procedimiento en cada una de las etapas de gestión de un curso con el empleo de la herramienta de $\mathrm{GC}^{\oplus}$. Fruto de esta experiencia se presentan los resultados obtenidos a lo largo de estos dos años y la muy positiva percepción por parte de los estudiantes que han participado este estudio; además de brindar información estadística y datos para la parametrización de cursos aplicados durante los últimos cinco semestres con el uso de la aplicación $\mathrm{GC}^{\circledast}$, se proporcionará como insumo de consulta para los Comités Académicos Institucionales y su respectivo análisis para la orientación de alternativas pedagógicas aplicables en modalidad presencial y a distancia en los diferentes programas académicos en las IES en Colombia, Latinoamérica y países hispanoparlantes, ampliamente adaptable a cualquier sistema educativo mundial. Por medio de ilustraciones y flujogramas se demostrará el diseño de los procedimientos necesarios, formatos compartidos como anexos y que son empleados para la creación, realización, seguimiento y evaluación en la gestión de un curso aplicando GC ${ }^{\circledast}$.

\section{PALABRAS CLAVE}

Experiencia educativa, Google Classroom ${ }^{\oplus}\left(\mathrm{GC}^{\circledast}\right)$, clase virtual, gestión pedagógica, docente universitario.

\section{ABSTRACT}

This research aims to demonstrate significant experience for more than two years in university teaching in classroom mode supported with the application of Google Classroom ${ }^{\oplus}\left(\mathrm{GC}^{\circledast}\right)$. It is considered as a case study to be included as an alternative and complementary learning methodology in the face-to-face mode and in virtualization in Higher Education Institutions (HEIs). The developed methodology consisted of the detailed design of a procedure in each of the management stages of a course with the use of the GC ${ }^{\circledast}$ tool. As a result of this experience, the results obtained throughout these two years and the very positive perception on the part of the students who have participated in the present courses are presented. This study, in addition to providing statistical information and data for the parameterization of courses applied during the last five semesters with the use of the $\mathrm{GC}^{\circledast}$ application, will provide input for consultation by the Institutional Academic Committees and their subsequent analysis for the orientation of alternatives. pedagogical courses applicable in person and remotely in the different academic programs in Colombia, Latin America and Spanish-speaking countries widely adaptable to any world educational system. Through illustrations and flowcharts, the design of the 
necessary procedures, shared formats as annexes and that are used for the creation, execution, monitoring and evaluation in the management of a course, will be demonstrated.

\section{KEYWORDS}

Educational experience, Google Classroom, virtual class, pedagogical management, university teacher

\section{INTRODUCCIÓN}

El uso de las TIC en la actualidad es parte importante en los fundamentos pedagógicos del proceso de aprendizaje, ya que estas contribuyen en el desarrollo del aprendizaje en las Instituciones de Educación Superior (IES) que ofrecen programas de pregrado y posgrado, acordes a las exigencias de alta calidad del Consejo Nacional de Acreditación de Colombia conforme a los lineamientos establecidos en el factor procesos académicos y componente (Uribe Urán, 2013).

Debido a los factores externos que afectan los modelos pedagógicos y las metodologías de aprendizaje institucionales implementadas en condiciones normales en la modalidad presencial y a raíz de diferentes crisis (Villacañas, 2013), como es el caso de la pandemia de COVID-19 (Presidencia de Colombia, 2020) que condujo al Aislamiento Preventivo Obligatorio decretado por el Ministerio de Educación Nacional (2020), se plantean las siguientes preguntas de investigación: ¿Qué alternativa existe para el manejo adecuado y eficaz de las clases presenciales, que contribuya a contrarrestar los efectos adversos en los métodos tradicionales de aprendizaje a nivel universitario en la IES? y ¿De qué manera se puede realizar gestión pedagógica de un curso aplicando modelos alternativos de aprendizaje en la modalidad presencial en los procesos metodológicos en las IES? Estos interrogantes serán resueltos en la conclusión de este caso de estudio que contribuye a la inclusión de estrategias metodológicas para la mejora de los procesos de planeación, desarrollo y evaluación curricular y como una opción de modelo alternativo de aprendizaje en la modalidad presencial en las IES.

\section{MARCO TEÓRICO}

A. Modelo de Acreditación en Alta Calidad para Instituciones y Programas Académicos. En Colombia, el Ministerio de Educación Nacional (MEN), el Consejo Nacional de Educación Superior (CESU) y el Consejo Nacional de Acreditación (CNA) durante un encuentro académico al que asistieron más de 500 rectores y directivos de Instituciones de Educación Superior del país para participar de una jornada de reflexión en torno a la calidad en la Educación Superior, socializaron los lineamientos para la actualización del Modelo de Acreditación en Alta Calidad para Instituciones y Programas.

La actualización se realiza sobre el artículo 28, indicando que los lineamientos para la Acreditación en Alta Calidad de los Programas Académicos son "aplicables en cualquiera sea las modalidades (presencial, a distancia, virtual, dual y sus combinaciones o integraciones) y niveles (técnico profesional, tecnológico, universitario, especialidad médico-quirúrgica, maestría y doctorado) (Ministerio de Educación, Consejo Nacional de Educación Superior y Consejo Nacional de Acreditación, 2020), y en su artículo 30, evaluación de la Alta Calidad de Programas Académicos, define doce factores que conforman el nuevo modelo de Acreditación de Alta Calidad en programas académicos universitarios colombianos. Para el enfoque del presente caso de investigación se destacan: Factor 5. Aspectos académicos y resultados de aprendizaje y Factor 10. Medios educativos y ambientes de aprendizaje. Por lo anterior, este documento pretende mostrar con resultados la manera como el diseño y gestión pedagógica por medio de $\mathrm{GC}^{\circledast}$ contribuirá en el cumplimiento de estas características y de los factores antes mencionados y extraídos del documento del CESU, para incluirlos como nuevas prácticas a nivel Institucional. 
B. Educación. La Real Academia Española define a la educación como "Instrucción por medio de la acción docente” (RAE, 2020). En el proceso de educación intervienen dos actores principales, el profesor y el estudiante. Por tal razón, en este documento se expone la relación y comunicación que será beneficiada entre estos dos, aplicando las secciones de comentarios en cursos en línea por medio de la plataforma GC ${ }^{\oplus}$.

C. Casos aplicados con Google Classroom ${ }^{\oplus}\left(\mathrm{GC}^{\oplus}\right)$. El uso de $\mathrm{GC}^{\circledast}$ se describió como un:

complemento basado en Tecnologías de la Información y la Comunicación (TIC), en capacitaciones presenciales a docentes de escuelas primarias. Se considera como caso de estudio a las instituciones de dicha modalidad, pertenecientes al Programa Integral para la Igualdad Educativa (PIIE) de la ciudad de Bahía Blanca, provincia de Buenos Aires, República Argentina. (Kraus, 2019)

En otro estudio de caso se examinaron aquellos factores que afectan la aceptación de los estudiantes a los cursos por $\mathrm{GC}^{\oplus}$ en Al Buraimi University College (BUC) en Omán (Al-Maroof, 2018), presentando como uno de los resultados sobresalientes la gran importancia para la toma de decisiones en las Instituciones Educativas y el hecho de que los estudiantes aplican sus clases en $\mathrm{GC}^{\circledast}$ por el respaldo de la tecnología y la facilidad de poder usarlo en el dispositivo móvil para aprovechar su sistema educativo.

D. Tecnologías de la Información y Comunicación-TIC. Las TIC han incidido ampliamente en la mejora de la productividad en general y se considera de suma importancia su ingreso en la educación con el objetivo de disminuir los índices de abandono escolar. Al respecto, Colina (2008) considera que el conjunto de servicios, redes, software y aparatos que integran las TIC están orientados a mejorar la calidad de vida de las personas dentro de un entorno $y$, por tal motivo, se acoplan a un sistema de información interconectado permitiendo romper las barreras que existen entre ellas. Por su parte, González Mariño (2008) define que la era digital ha generado grandes transformaciones y ventajas con respecto al ámbito educativo.

El autor del presente caso de investigación en su tesis de maestría señala la importancia de que desde en las IES se implementen estrategias pedagógicas con el uso de las TIC, recomendando que:

la preocupación de la academia en cuanto a mejorar las condiciones actuales de dedicación de tiempo en los estudiantes para el autoaprendizaje e investigación, se pueden aminorar implementando políticas y estrategias pedagógicas que involucren modelos de aprendizaje mediados por TIC, por esta razón, los esfuerzos y recursos que se inviertan en este fin, serán beneficiosos a corto plazo. (Medaglia, 2015)

El empleo de la aplicación $\mathrm{GC}^{\circledast}$ para actividades académicas se ha ido incrementando masivamente a nivel mundial en los diferentes niveles de la educación, según lo registran los datos recientemente publicados por el diario mexicano en economista “...en la plataforma de aplicaciones para dispositivos, Android (Play Store) es la tercera con el mayor número de descargas en el mundo en la categoría de aquellas gratuitas, sólo después de Zoom y Google Classroom " (Aja, 2020).

Debido a la pandemia Coronavirus (COVID-19), a nivel global el incremento en número de estudiantes y profesores que se han sumado al uso de la aplicación de $\mathrm{GC}^{\varpi}$ se "ha duplicado desde principios de marzo de 2020 y en la actualidad cuenta con cien millones de usuarios", según publicó el diario La Voz ( La Voz, 2020).

\section{METODOLOGÍA}

La metodología empleada consistió en identificar aquellos factores fundamentales a tener en cuenta para el desarrollo y puesta en escena de un curso de 
asignatura y su diseño estructural desde la aplicación de GC $^{\oplus}$, para diseñar de manera detallada los procedimientos en las etapas de gestión de un curso.

\section{RESULTADOS Y CONCLUSIONES}

Por medio de gráficos, ilustraciones, formatos y guías se ilustrarán los procedimientos establecidos para la creación, diseño de estructura, alojamiento de material de consulta, documentos, archivos de trabajo y gestión de notas de un curso con la aplicación GC ${ }^{\circledast}$. A su vez, se compartirán las soluciones realizadas a los problemas más comunes en la administración del curso.

Para facilitar la comprensión de la herramienta se debe tener en cuenta algunas consideraciones para el antes, durante y después de un curso. En este sentido, se identificaron cinco etapas de gestión de una clase desde la aplicación GC $\mathrm{C}^{\circledast}$. A continuación, se describen los argumentos en cada una de ellas:

A. Requerimientos básicos para acceso a la plataforma $\mathrm{GC}^{\oplus}$ y creación de la clase. Esta etapa abarca desde la creación o uso de la cuenta de correo Gmail hasta la creación del curso. Para el uso de la aplicación académica de $\mathrm{GC}^{\circledast}$ es necesario contar con una cuenta de correo electrónico de $\mathrm{Gmail}^{\circledR}$ tanto para el(los) profesor(es) como los estudiantes participantes. Desde la cuenta del correo Gmail se accede a las aplicaciones de Google y se selecciona $\mathrm{GC}^{\circledast}$. El procedimiento correspondiente se presenta en el flujograma de la Figura 1:

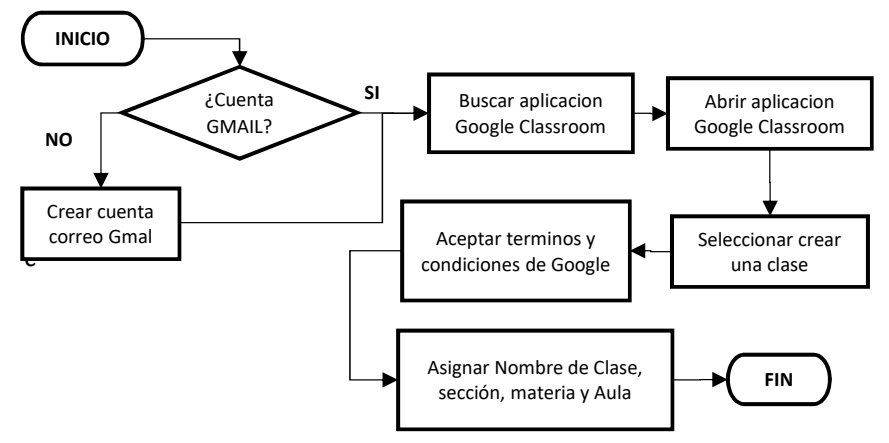

Fig. 1. Procedimiento creación de curso en $\mathrm{GC}^{\circledast}$. Fuente: Elaboración propia.
En la sección Crear una clase se recomienda diligenciar la siguiente información solicitada por el formulario en línea, así:

- $\quad$ Nombre de Clase: Nombre de la asignatura del curso a impartir

- Sección: Grupo del curso asignado internamente por la Institución Educativa

- Materia: Descripción del Programa Académico

- Aula: Código de la asignatura

B. Estructuración de las secciones y contenidos del curso. El alcance de esta etapa inicia con el acceso al muro transaccional de Trabajo de clase y abarca hasta la publicación de material, tareas o preguntas del curso. Creado el curso en la aplicación de $\mathrm{GC}^{\circledast}$ es imperativo conocer las secciones o muros que el curso contiene, dado que cada sección tiene ciertas condiciones a tener presente en el proceso de constituir los contenidos del curso. Por defecto el curso está compuesto de cuatro secciones o muros que tienen las siguientes propiedades:

- $\quad$ Muro de Tablón o Novedades: en esta sección se muestran cada una de las publicaciones de manera automática, de consulta más reciente hasta las más antiguas. En esta sección se incluyen y publican mensajes, noticias, y se comparten archivos en cualquier formato, enlaces de páginas web, videos y formularios en línea, entre otros, de manera inmediata o programada; permite reutilizar publicaciones anteriores para su actualización con datos e información reciente. Por cada publicación emitida, se permite incluir comentarios por parte de los estudiantes inscritos al curso y del (los) profesor(es). Esta sección es de consulta de información y acceso directo a los temas creados en el muro de Trabajo en Clase.

- $\quad$ Muro de Trabajo en Clase: sección de transferencia de información y por el cual se facilita la creación de actividades tipo talleres, trabajos, cuestionarios, 
evaluaciones, contenido de las unidades, temas de la asignatura, calificación de los compromisos académicos realizados por los estudiantes, emitir reporte de notas y organizar la clase por módulos o temas. Esta sección es transaccional para la asignación de temas, actividades académicas, registro de notas y respuesta a los comentarios publicados por los alumnos y comunicaciones en público o privado con los estudiantes.

- $\quad$ Muro de Personas: sección donde se registran los profesores y alumnos que han ingresado a la clase por medio del acceso a la aplicación registrando el código de la clase único, que se asigna de manera inmediata y posterior a su creación (suministrado por el sistema al profesor previamente). También está habilitado el proceso de invitar a profesores o estudiantes, siempre y cuando se cuente con el (los) correos electrónicos Gmail de los estudiantes o profesores. En esta sección es posible enviar correos electrónicos masivos o individuales según sea el caso.

- Muro de Calificaciones: al vincular estudiantes al curso y asignar tareas, trabajos o actividades para evaluar, en esta sección se describen la fecha y descripción del compromiso académico y el estado por cada estudiante de cumplimiento en la entrega o no de cada compromiso académico. Posterior a la evaluación y registro de las notas por cada estudiante y cada actividad de aprendizaje, se facilita la descarga de copia de las notas por actividad de aprendizaje o todas en archivo de Excel para su consulta o edición posterior.

Desde el muro de Trabajo en Clase se crean los contenidos del curso y se clasifica el material, y contenidos por módulos o temáticas. En la Figura 2 se presenta el procedimiento correspondiente.

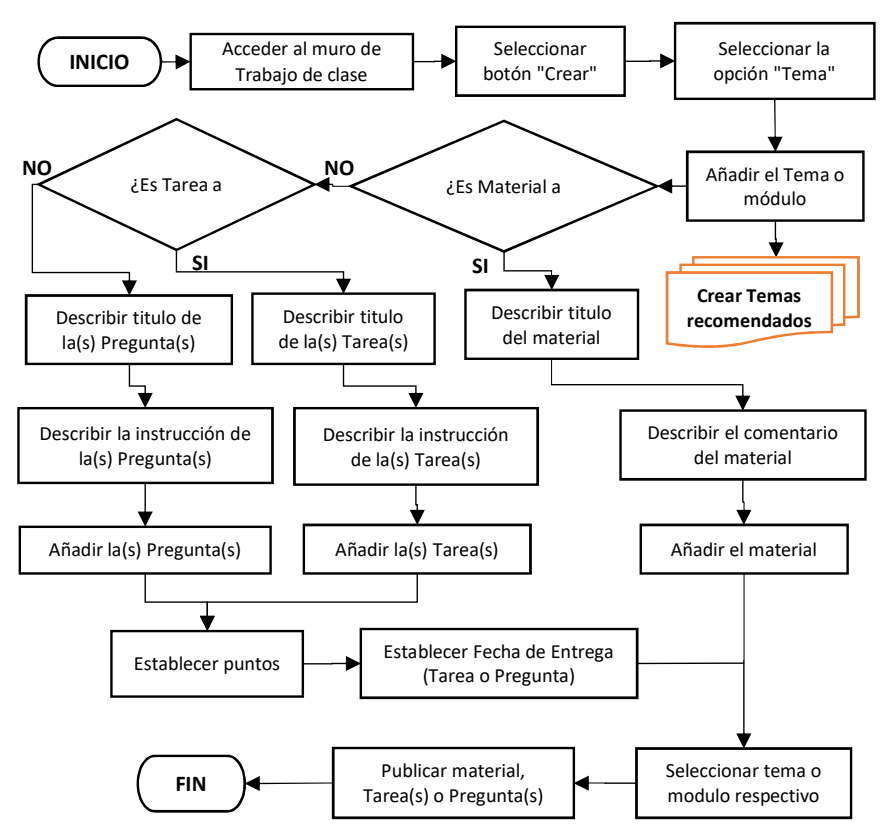

Fig. 2. Procedimiento para Estructuración de las secciones y contenidos del curso en $\mathrm{GC}^{\circledR}$. Fuente: Elaboración propia.

Con referencia a los temas que se deberán establecer para parametrizar en cada clase y en adelante, se recomienda como mínimo los siguientes módulos

- $\quad$ Información General
- $\quad$ Contenido Programático
- $\quad$ Actividades de Aprendizaje
- $\quad$ Parciales
- Proyecto integrador
- $\quad$ Presaberes
- $\quad$ Consultas Bibliográficas
- $\quad$ Preguntas e Inquietudes

En la Tabla 1 se describe de manera detallada cada una de las relaciones de los temas o módulos propuestos, su propósito en la parametrización y tipos de documento e información a parametrizar en cada clase. 


\begin{tabular}{|c|c|c|}
\hline $\begin{array}{l}\text { Temas o Modulos } \\
\text { Sugeridos }\end{array}$ & Propósito Del Tema O Módulo & Crear / Título / Contenido / Adjunto \\
\hline Información General & $\begin{array}{l}\text { Módulo para la publicación de saludo de bienvenida a los } \\
\text { estudiantes del curso. }\end{array}$ & Material / Bienvenida / Descripción en texto del saludo de Bienvenida / Ninguno Adjunto. \\
\hline Contenido Programático & $\begin{array}{l}\text { Módulo para la publicación del Calendario Académico y } \\
\text { Acuerdo Pedagógico incluyendo Cronograma con fechas } \\
\text { propuestas de entrega de Actividades de Aprendizaje y } \\
\text { Parciales(de acuerdo con las fechas presentadas en la } \\
\text { resolución emitida desde la rectoría de la Institución } \\
\text { Universitaria). }\end{array}$ & $\begin{array}{l}\text { Material / Resolución / Descripción en texto "Número de Resolución vigente" / Adjuntar PDF Resolución. } \\
\text { Material / Acuerdo Pedagógico / Descripción en texto "Acuerdo Pedagógico" / Adjuntar archivo "Acuerdo } \\
\text { Pedagógico de la Asignatura_periodo 202X-A/B". } \\
\text { Material / Cronograma Académico / Descripción en texto "Cronograma Académico" / Adjuntar archivo } \\
\text { "Cronograma Asignatura periodo 202X-A/B". }\end{array}$ \\
\hline Contenido Unidades & $\begin{array}{l}\text { Módulo donde se consultan cada uno de las temáticas de } \\
\text { la asignatura presentados por separado por Unidades o } \\
\text { Capítulos. }\end{array}$ & $\begin{array}{l}\text { Material* / Unidad \#* y Descripción del Tema de la Unidad \# / Descripción en texto de subtemas de la Unidad \& } \\
\text { Contenido de la unidad (presentaciones PPT, Lectura de libro, Ejercicios en clase, etc.) } \\
\text { *Se repite para el número de temas, unidades y material(contenido por unidad) a presentar en la asignatura } \\
\text { durante el periodo académico vigente. }\end{array}$ \\
\hline Actividades de Aprendizaje & $\begin{array}{l}\text { Módulo de consulta y respuesta a los compromisos } \\
\text { académicos como talleres, quizes, trabajos, cuestionarios } \\
\text { establecidas en el Acuerdo Pedagógico de la asignatura. }\end{array}$ & $\begin{array}{l}\text { Tarea o Pregunta** / Actividad de Aprendizaje \#** / Descripción en texto de la instrucción o propósito del } \\
\text { compromiso académico por realizar, especificando fecha de entrega y condiciones para la entrega / Adjuntar } \\
\text { archivo o enlace de la Actividad de aprendizaje por desarrollar. } \\
* * \text { Se repite para el número de Actividades de Aprendizaje, Tareas a presentar según el Acuerdo Pedagógico } \\
\text { durante el periodo académico vigente. }\end{array}$ \\
\hline
\end{tabular}

\begin{tabular}{|c|c|c|}
\hline Parciales & $\begin{array}{l}\text { Módulo para la consulta y realización de las evaluaciones } \\
\text { establecidas en el Acuerdo Pedagógico de la asignatura. }\end{array}$ & $\begin{array}{l}\text { Tarea o Pregunta / Parcial I / Descripción en texto de la instrucción en los temas a evaluar, método de evaluar, } \\
\text { medio de desarrollo y mecanismo para la entrega de la evaluación 1, especificando fecha de entrega y porcentaje } \\
\text { de la nota(20\%) con respecto a la nota definitiva / Adjuntar archivo o enlace de la Evaluación } 1 \text { o Parcial } 1 . \\
\text { Tarea o Pregunta / Parcial II / Descripción en texto de los temas a evaluar, método de evaluar, medio de desarrollo } \\
\text { y mecanismo para la entrega de la evaluación 2, especificando fecha de entrega y porcentaje de la nota(20\%) con } \\
\text { respecto a la nota definitiva / Adjuntar archivo o enlace de la Evaluación } 2 \text { o Parcial } 2 . \\
\text { Tarea o Pregunta / Parcial Final / Descripción en texto de los temas a evaluar, método de evaluar, medio de } \\
\text { desarrollo y mecanismo para la entrega de la evaluación final, especificando fecha de entrega y porcentaje de la } \\
\text { nota(25\%) con respecto a la nota definitiva / Adjuntar archivo o enlace de la Evaluación Final o Parcial Final. }\end{array}$ \\
\hline Proyecto integrador & $\begin{array}{l}\text { Módulo para la consulta y realización del Proyecto } \\
\text { Integrador de la asignatura y establecido en el Acuerdo } \\
\text { Pedagógico de la asignatura. }\end{array}$ & $\begin{array}{l}\text { Tarea / Proyecto Integrador / Descripción en texto de los temas para contenidas en el proyecto integrador, } \\
\text { asignaturas que serán incluidas en el desarrollo del trabajo y mecanismo para la entrega del trabajo del Proyecto, } \\
\text { especificando fecha de entrega y porcentaje de la nota( } 20 \% \text { con respecto a la nota definitiva / Adjuntar archivo } \\
\text { modelo y estructura del Proyecto Integrador. }\end{array}$ \\
\hline Presaberes & $\begin{array}{l}\text { Módulo para la consulta de extracto de textos o } \\
\text { preguntas de consulta previas al desarrollo de cada clase } \\
\text { o temática a ser presentada en las siguientes clases. }\end{array}$ & $\begin{array}{l}\text { Tarea o Pregunta*** / Presaber de la Temática o Unidad*** / Descripción en texto de la instrucción o propósito de } \\
\text { la consulta previa a la clase o temas a presentar próximamente / Adjuntar archivo, enlace o cuestionario de } \\
\text { preguntas relacionada con el tema a presentarse en clase. } \\
* * * \text { Se repite para el número de temáticas o Unidad, Tareas o preguntas consultadas previamente a la clase por } \\
\text { presentar (estas no son calificables si lo considera el profesor). }\end{array}$ \\
\hline Consultas Bibliográficas & $\begin{array}{l}\text { Módulo para la consulta permanente de libros digitales, } \\
\text { link de las base de datos con textos digitales, artículos o } \\
\text { revistas (referencias bibliográficas) con relación al área } \\
\text { del conocimiento o temática de la asignatura. }\end{array}$ & $\begin{array}{l}\text { Material / Titulo del texto, libro o articulo / Descripción en texto "Titulo del material compartido_" / Adjuntar PDF } \\
\text { Libro, articulo, texto, o link de acceso a la biblioteca digital, etc. } \\
\text { De acuerdo con el material a compartir o link de acceso a la biblioteca digital de la Institución, se publicará el } \\
\text { material o link correspondiente. }\end{array}$ \\
\hline Web site Complementario & $\begin{array}{l}\text { Módulo para la consulta permanente de sitios web, de } \\
\text { paginas de internet científicamente válidas, blogs } \\
\text { relacionados con el área del conocimiento o temática de } \\
\text { la asignatura. }\end{array}$ & $\begin{array}{l}\text { Material / Consulta de de sitios Web / Descripción en texto del Web Site a consultar / Adjuntar ruta URL por el link } \\
\text { de acceso por Internet. } \\
\text { De acuerdo con la cantidad de sitios web validados se comparte el link de acceso por Internet. }\end{array}$ \\
\hline Preguntas e Inquietudes & $\begin{array}{l}\text { Módulo para formular preguntas e inquietudes con } \\
\text { relación al curso, dudas técnicas con la aplicación. }\end{array}$ & $\begin{array}{l}\text { Material / Preguntas e Inquietudes / Descripción en texto describiendo el propósito de la seccción para aclarar } \\
\text { dudas funcionales a cerca de la aplicación entre otros/ Ninguno Adjunto. }\end{array}$ \\
\hline
\end{tabular}

Tabla 1. Relación de Temas, su propósito y tipos de documento a publicar en el curso. Fuente: Elaboración propia.

C. Procesamiento para la elaboración de actividades de aprendizaje, evaluaciones, mensajes informativos y comunicación con los estudiantes participantes del curso. Etapa que abarca la asignación de material, tareas o preguntas en cada tema o módulo diseñado hasta la publicación de cada uno en el curso o clase. Este proceso ha sido definido en la Tabla 1, columna tres (crear Título, Contenido y Adjunto).
Para el proceso del cargue de los materiales, tareas o preguntas, este ha sido descrito en la Figura 2, en el flujograma de creación de temas, materiales o tareas, y para la preparación de actividades de aprendizaje se han empleado formatos diseñados para estandarizar su uso. Los formatos empleados para la presentación del Acuerdo Pedagógico, Ficha y Desarrollo de las Actividades de Aprendizaje se muestran a continuación en las figuras 3, 4, 5 y 6, respectivamente: 


\begin{tabular}{|c|c|c|}
\hline \multirow{3}{*}{ 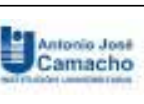 } & \multirow{2}{*}{\multicolumn{2}{|c|}{ Facultad de Ingeniería }} \\
\hline & & \\
\hline & Programa Ingeniería Industrial & Página 1 | 2 \\
\hline
\end{tabular}

CALENDARIO 2020-1 - UNIAIC CALENDARIOA A VIERNES 18:30-21:30 Resolucion 022 de 25/10/2019 - UNIAIC

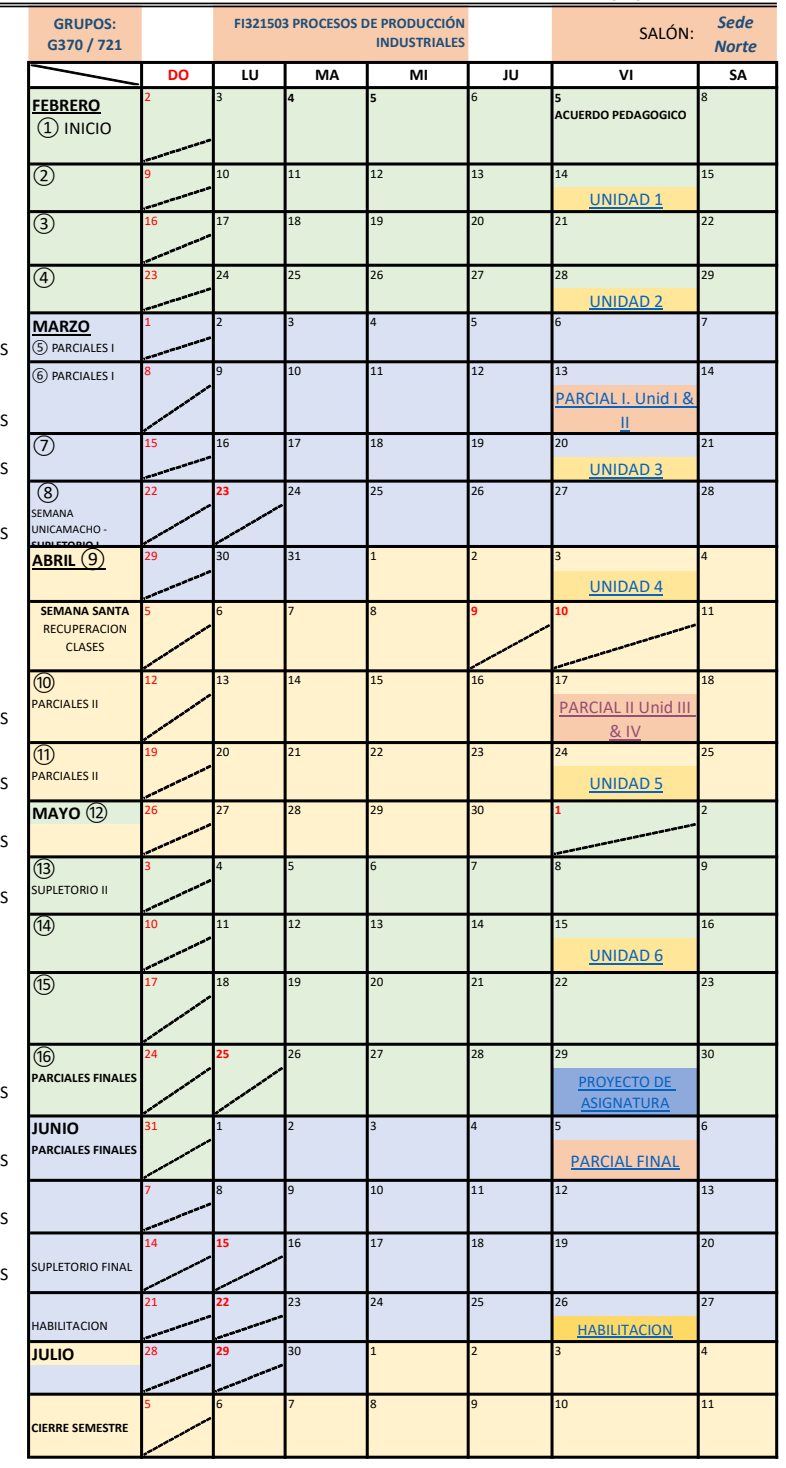

CONTENIDO DE LA ASIGNATURA -

FI321503 PROCESOS DE PRODUCCIÓN INDUSTRIALES

Unidad I. Fundamentos de Procesos Industriales

Unidad II. Gestión de la Producción y las Operaciones

Unidad III. Procesos Industriales I

Unidad V. Control y Evaluación de la Produccion

Unidad VI. Mejora y Automatización de procesos Industiales

ESTRUCTURA DE NOTAS DE LA ASIGNATURA: $\quad$ FI321503 PROCESOS DE PRODUCCIÓN INDUSTRIALES

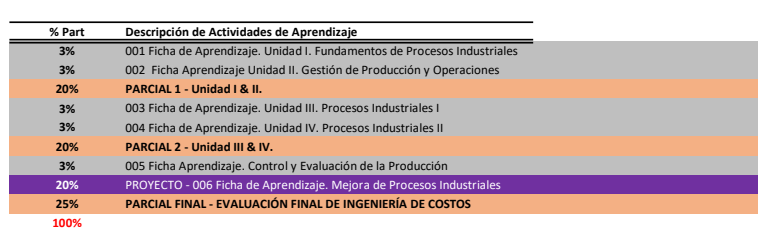

Mg. Ing. Oswaldo Medaglia Zapata

Fig. 3. Formato propuesto para Acuerdos Pedagógicos en el curso.

Fuente: Elaboración propia.
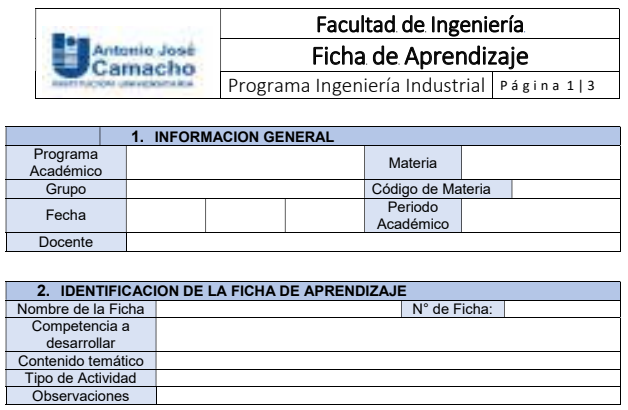

\begin{tabular}{|l|l|l|}
\hline 3. ACTIVIDADES A DESARROLLAR \\
\hline $\begin{array}{l}\text { Actividad de } \\
\text { aprendizaje } 1\end{array}$ \\
\hline Descripción & \multicolumn{3}{l|}{} \\
\hline $\begin{array}{c}\text { Resultado de } \\
\text { Aprendizaje }\end{array}$ & $\begin{array}{c}\text { Medio de } \\
\text { Entrega }\end{array}$ & \\
\hline Fecha de Entrega & $\begin{array}{c}\text { Criterio de } \\
\text { Evaluación }\end{array}$ & Ver numeral 5. Rubrica \\
\hline Criterio de entrega & & \\
\hline \% Evaluación & & \\
\hline
\end{tabular}

\begin{tabular}{|c|c|c|}
\hline $\begin{array}{c}\text { Actividad de } \\
\text { aprendizaje 2 }\end{array}$ & \multicolumn{3}{|l|}{} \\
\hline Descripción & \multicolumn{3}{|l|}{} \\
\hline $\begin{array}{c}\text { Resultado de } \\
\text { Aprendizaje }\end{array}$ & \multicolumn{3}{|l|}{} \\
\hline Fecha de Entrega & $\begin{array}{c}\text { Medio de } \\
\text { Entrega }\end{array}$ \\
\hline Criterio de entrega & $\begin{array}{c}\text { Criterio de } \\
\text { Evaluación }\end{array}$ & Ver numeral 5. Rubrica \\
\hline \% Evaluación & & \\
\hline
\end{tabular}

$2017 \quad$ Ing. Oswaldo Medaglia Zapato

Fig. 4. Formato de Ficha de Aprendizaje Fuente: Elaboración propia.

\begin{tabular}{|c|c|c|}
\hline \multirow{3}{*}{$\begin{array}{l}\text { Feflanto Jost } \\
\text { Camacho }\end{array}$} & \multirow{2}{*}{\multicolumn{2}{|c|}{$\begin{array}{c}\text { Facultad de Ingeniería } \\
\text { Desarrollo Actividad de Aprendizaje }\end{array}$}} \\
\hline & & \\
\hline & Programa Ingeniería Industrial & Página $1 / 2$ \\
\hline
\end{tabular}

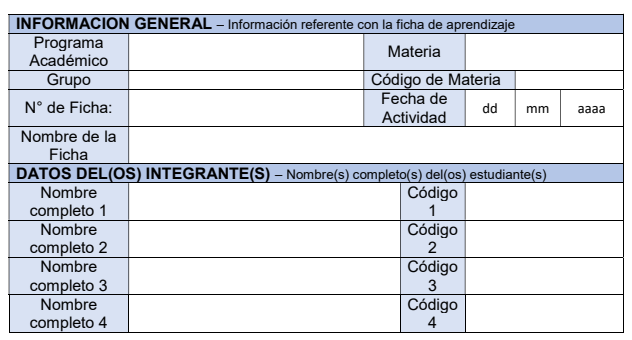

INTRODUCCION - Información de la descripción de la actividad a desarrollar en la ficha de aprendizaje
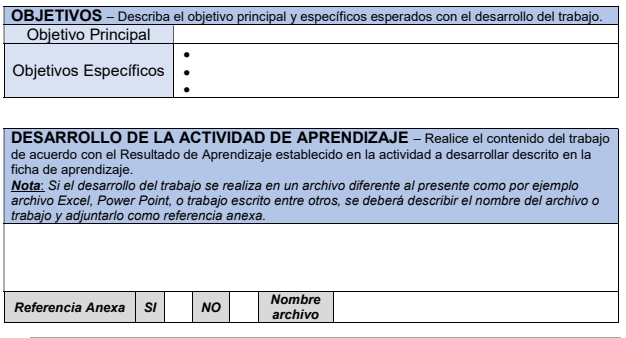

2017

Ing. Oswaldo Medaglia Zapata

Fig. 5. Formato Word Actividad de Aprendizaje Fuente: Elaboración propia. 


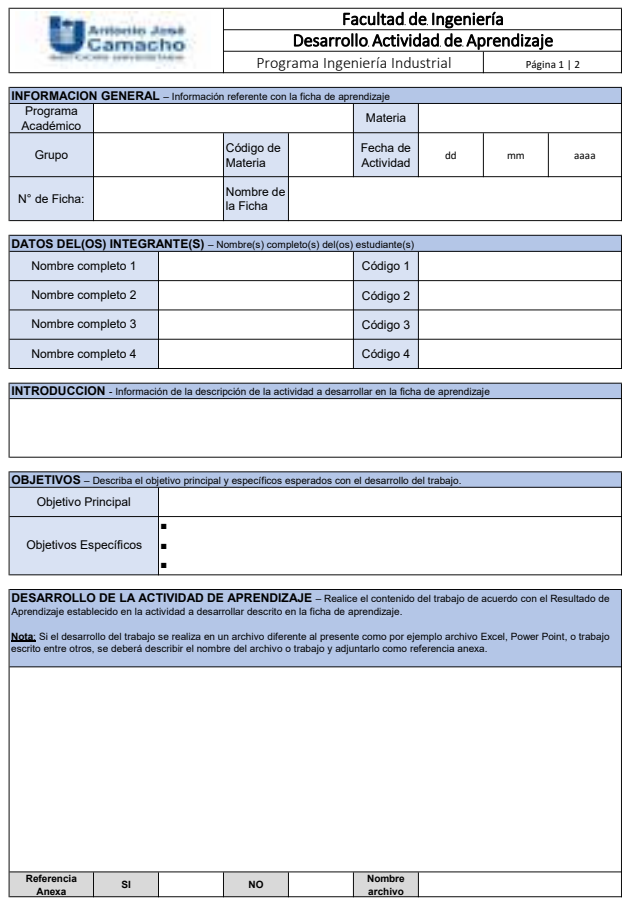

Cada uno de los anteriores formatos tienen el propósito de unificar los documentos y normalizar el desarrollo y entrega de las actividades de aprendizaje por parte de los estudiantes, así como la recepción para el proceso de evaluación de los compromisos académicos por parte del(los) profesor(es) de la asignatura.

Otro mecanismo para subir actividades y facilitar el desarrollo y evaluación de las tareas o trabajos, es diseñar formularios en línea con el fin de que, al acceder a uno de ellos por medio de un vínculo, el formulario sea diligenciado en línea y al finalizar se envía, sin necesidad de adjuntar archivos en la plataforma. Formularios como los que se muestran en la Figura 7:

Fig. 6. Formato Excel Actividad de Aprendizaje Fuente: Elaboración propia.

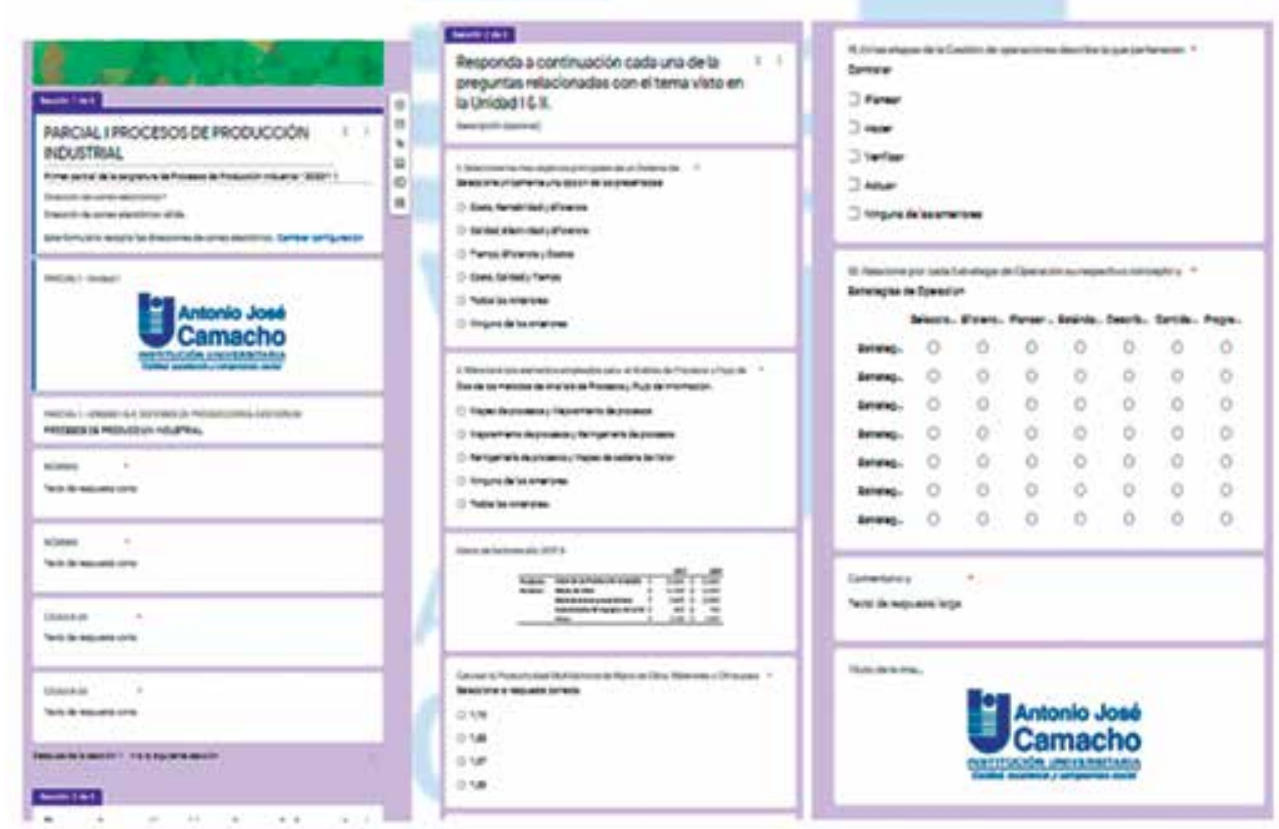

Fig. 7. Formulario Tipo para la creación de Parciales en línea, con calificación inmediata. Fuente: Elaboración propia.

El enlace para acceder al anterior formulario en línea es: https://forms.gle/rk5QkNmGNmwrnzG28 En cuanto a los mensajes informativos por cada tema o módulo establecido, se realiza desde la sección inferior del contenido, en el espacio "incluir comentario" y con las modalidades de publicación pública o privada, según la elección (Figura 8). 

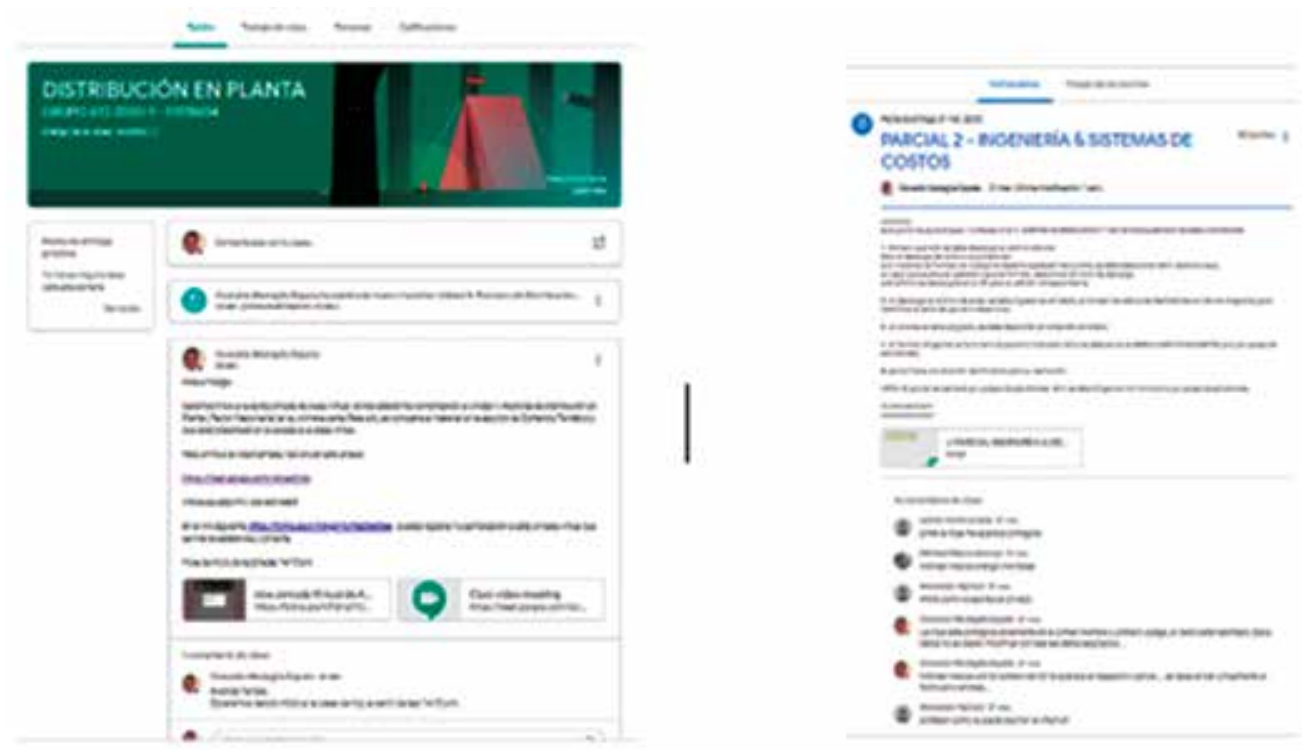

Fig. 8. Demostración desde dos cursos de la mensajería con los estudiantes.

Fuente: Elaboración propia.

La comunicación con los estudiantes participantes del curso es permanente, ya que en cada sección creada se despliega en la parte inferior el espacio de comentarios de clase, facilitando la trasmisión de la información entre estudiantes-docente y viceversa. En el numeral E. Funcionalidad del curso desde la aplicación móvil, se evidenciará la facilidad de la mensajería desde cualquier tipo de smartphone, celular o tableta al descargar la App de $\mathrm{GC}^{\circledR}$ en cualquiera de los dispositivos móviles con acceso a internet.

D. Calificación de actividades de aprendizaje y evaluaciones, generación de reporte de notas y revisión del estado de las notas del curso. El alcance de esta etapa de gestión tiene su inicio con el ingreso en el muro transaccional de Trabajos de clase, incluyendo los flujos necesarios para el manejo de Actividades de Aprendizaje y evaluaciones hasta la publicación de notas por cada uno de los participantes de la clase. En la Figura 9 se presenta el flujograma correspondiente al procedimiento.

E. Funcionalidad del curso desde la aplicación móvil. En esta consideración se contempla la operatividad de la clase desde dispositivos móviles y tabletas digitales con acceso de Internet. Para acceder desde un smartphone, celular móvil o tableta es necesario acceder a la tienda de aplicaciones digitales de acuerdo con el sistema operativo, sea Android o IOS, descargar, instalar y acceder a la clase de GC ${ }^{\circledast}$. En el flujograma de la Figura 10 se especifica el paso a paso para configuración en el dispositivo de una clase en $\mathrm{GC}^{\oplus}$.

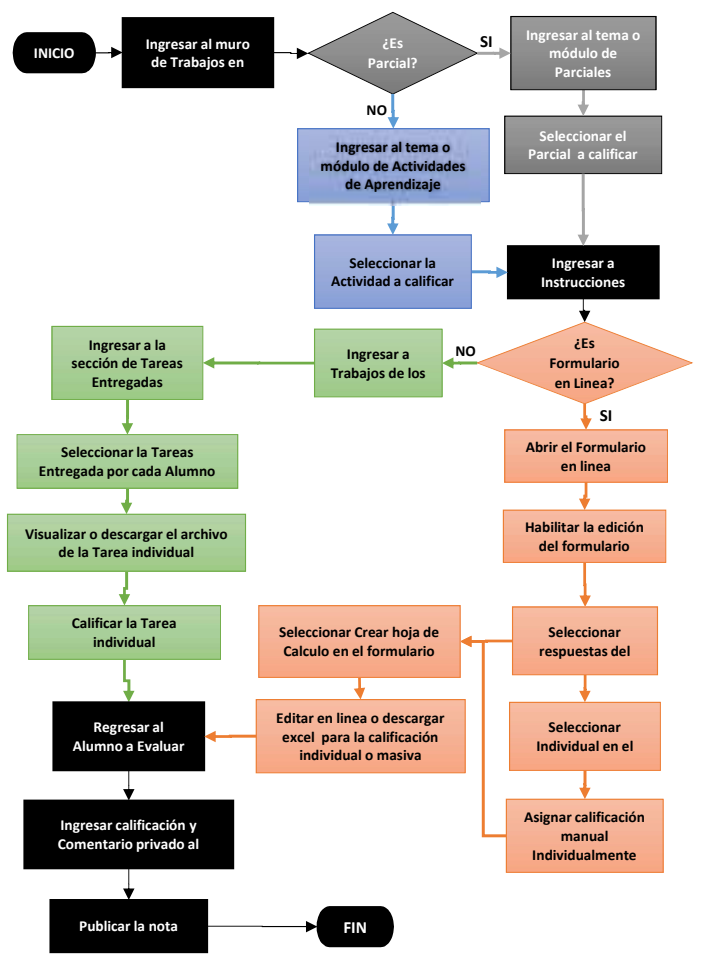

Fig. 9. Procedimiento subir Actividades de Aprendizaje, Parciales o formularios en línea y publicación de notas del curso en GC Fuente: Elaboración propia. 
En flujograma de la Figura 9, se representan los diferentes flujos a seguir así:

$\rightarrow$ Flujo transaccional desde el muro de Trabajos en Clase de Google Classroom.

$\rightarrow$ Flujo para manejo de Actividades de Aprendizaje en Trabajo de Clase.

$\longrightarrow$ Flujo para manejo de Parciales en Trabajo de Clase

$\longrightarrow$ Flujo para manejo de formularios en línea como material entregado en Clase.

$\longrightarrow$ Flujo para manejo de archivos como material entregado en Tabla de Clase.

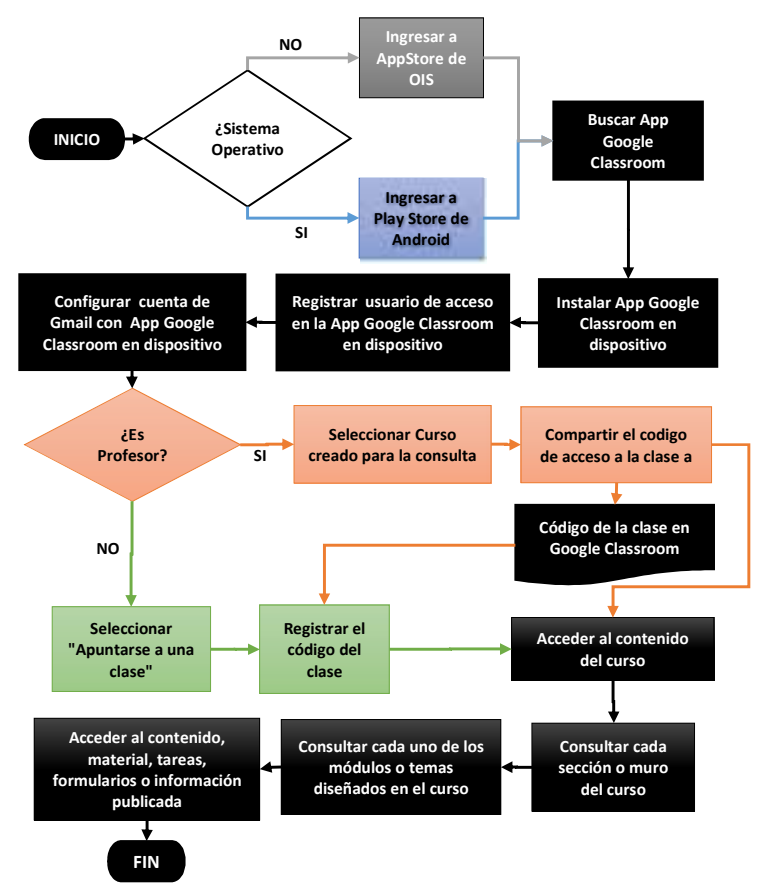

Fig. 10. Procedimiento para el acceso al curso en $\mathrm{GC}^{\circledast}$ desde dispositivos móviles. Fuente: Elaboración propia.

En el flujograma de la Figura 10 se representan los diferentes flujos o pasos a seguir según:

$\rightarrow$ Flujo transaccional desde el muro de Trabajos en Clase de Google Classroom.

$\longrightarrow$ Flujo para manejo desde sistema operativo Android.

$\longrightarrow$ Flujo para manejo desde sistema operativo OIS $\longrightarrow$ Flujo desde el perfil como profesor.

$\longrightarrow$ Flujo desde el perfil de alumno.
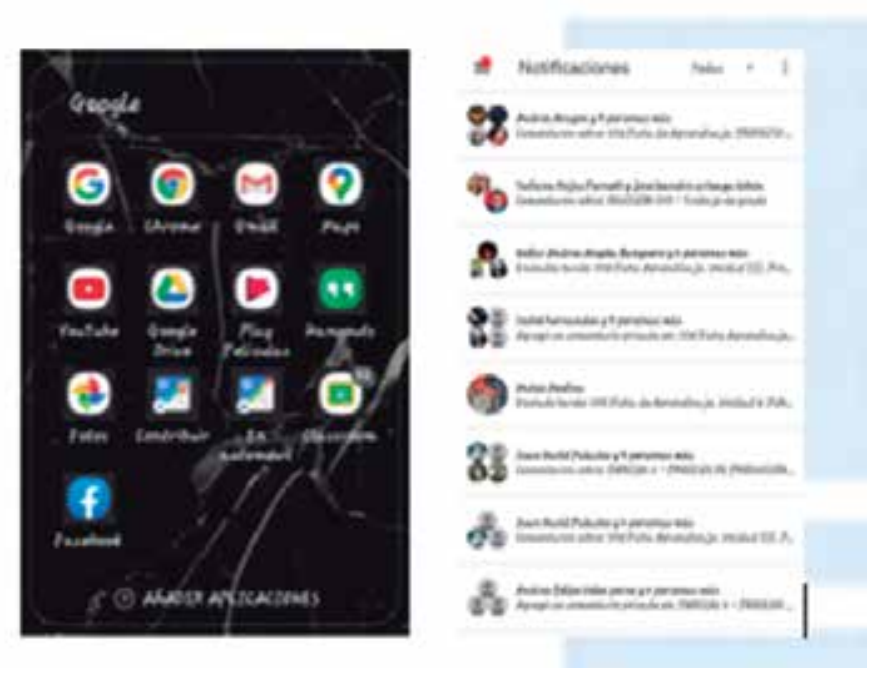

Fig. 11. . Demostración de visualización de acceso a $\mathrm{GC}^{\circledast}$ desde dispositivos móviles.

Fuente: Elaboración propia.

En la Figura 10 se aprecian las visualizaciones desde un dispositivo móvil, accediendo al contenido del curso, de la misma forma como se puede realizar desde la cuenta de correo Gmail y la aplicación de $\mathrm{GC}^{\circledast}$ del equipo de cómputo de escritorio o PC portátil.

*Este correo electrónico ha sido utilizado para creación, gestión pedagógica y recepción de notificaciones de las clases en GC: oswaldo.medaglia@gmail.com. .

A continuación, se presentan algunos de los resultados obtenidos en el desarrollo de más de dos años de experiencia en la implementación de clases para algunas de las asignaturas impartidas en la Facultad de Ingenierías para los programas académicos de Ingeniería Industrial y Tecnología de Producción Industrial en la Institución Universitaria José Antonio Camacho como apoyo a las clases presenciales. Desde el primer encuentro con los estudiantes de cada asignatura presencial y durante la presentación del curso se les facilita el código de acceso e ingreso a la clase disponible en $\mathrm{GC}^{\oplus}$, proporcionando toda la instrucción necesaria para el acceso, manejo y conocimiento del curso desde $\mathrm{GC}^{\circledR}$. 


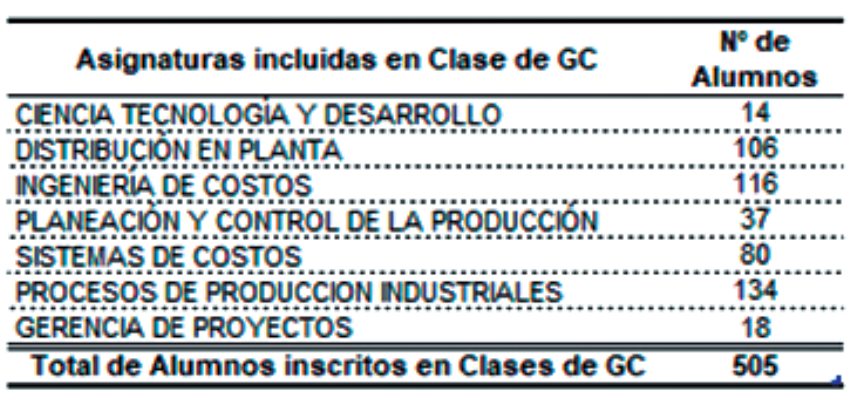

Tabla 2. Asignaturas diseñadas en GC entre 2018-2020(A). Fuente: Elaboración propia.

Numero de alumnos inscritos en cursos por asignatura en Google Classroom entre 2018 - 2020(A)

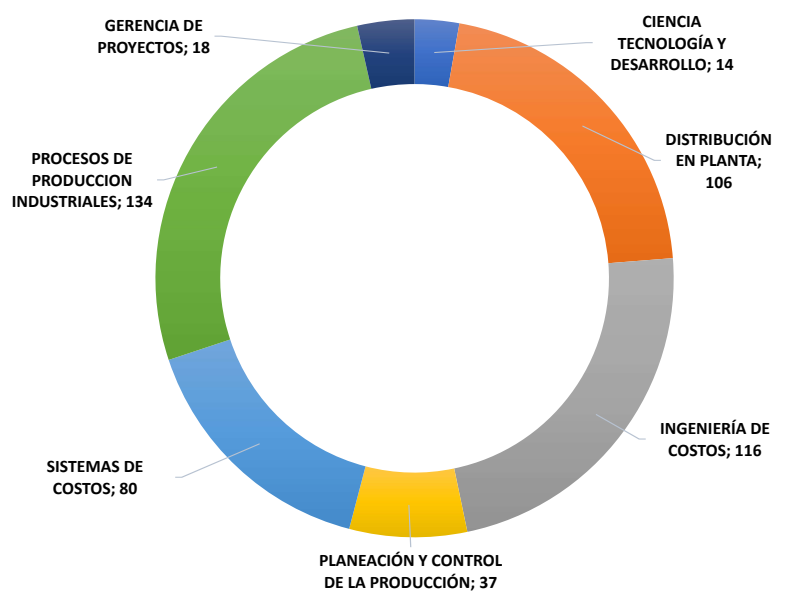

Fig. 12. Alumnos inscritos por asignatura en GC. Fuente: Elaboración propia.

El presente estudio de caso de investigación abarca las clases realizadas desde el año 2018 hasta 2020 (A) primer semestre, de los cuales 505 alumnos han participado activamente en alguno de los cursos diseñados en la aplicación de $\mathrm{GC}^{\oplus}$ para las 8 asignaturas del programa de Ingeniería Industrial y Tecnología de Producción Industrial, según se demuestra en la Tabla 2.

Los resultados presentados en la Figura 12 muestran que la mayor cantidad de estudiantes inscritos a los cursos diseñados en $\mathrm{GC}^{\circledast}$, corresponden a las asignaturas de Ingeniería de Costos y Sistemas de Costos (esta asignatura ha sido fusionada por grupos de los Programas de Ingeniería y Tecnología debido a la homologación en los contenidos de las asignaturas en ambos programas académicos) con un total de
39\% de participantes activos, Procesos de Producción Industrial con el 27\% y Distribución en Planta con el $21 \%$ de asistencia al curso en la aplicación. La participación por Programa Académico, según lo demuestra en la Figura 13, la mayor participación de alumnos está en Tecnología en Producción Industrial con el $55 \%$ e Ingeniería Industrial con el $45 \%$.

\begin{tabular}{|c|c|c|c|c|}
\hline Asignatura incluida en Clases en GC & Ano 2018 & Ano 2019 & Ano 2020 & Total \\
\hline DSTRBUCION EN PLANTA & 2 & 2 & 1 & 5 \\
\hline NGENERIA OE COSTOS & 2 & 1 & & 3 \\
\hline SSTENAS DE COSTOS & 2 & 1 & & 3 \\
\hline NGENERAA OE COSTOS \& SSTEUAS OE COSTOS & & "i" & 1 & '2' \\
\hline PLANACON Y CONTROL OC LAPROOUCCION & 2 & 2 & 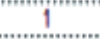 & "s" \\
\hline PROCESOS DE PROOUCCON NOUSTRLALS & 1 & 4 & 2 & 7 \\
\hline CENCA TECNOLOGA YOESARROLLO & & i & & 1 \\
\hline GERENCA DE PROYECTOS & & 1 & & 1 \\
\hline DRECCOON DE PROYECTO DE GRADO & 1 & 4 & 1 & 6 \\
\hline Total de Clases diseñadas en GC por Año & 10 & 17 & 6 & 33 \\
\hline
\end{tabular}

Tabla 3. Relación de cantidad en GC por asignatura diseñados entre 2018 - 2020 Fuente: Elaboración propia.

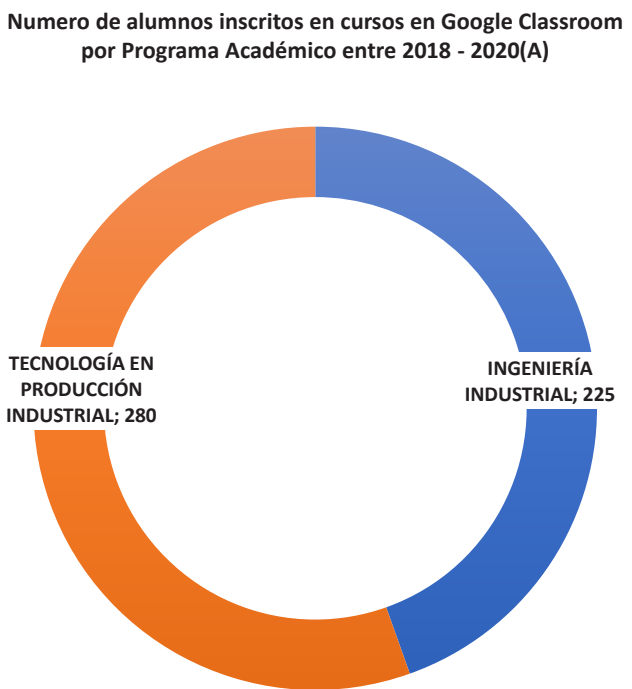

Fig. 13. Número de alumnos en GC por Programa Académico. Fuente: Elaboración propia.

En la Tabla 3 se muestra la cantidad de cursos diseñados e incluidos en la aplicación $\mathrm{GC}^{\circledast}$ por año. El promedio anual es de 13 clases gestionadas desde GC ${ }^{\circledast}$. Es importante anotar que la asignatura de Dirección de trabajo de grado también se ha incluido en $\mathrm{GC}^{\circledast}$, toda vez que las jornadas de acompañamiento, asesorías, 
revisiones de avances de los trabajos y correcciones, así A continuación, se muestran los resultados cualitativos como la comunicación con los aspirantes a graduación de la aplicación de los cursos diseñados desde la misma se hacen desde la sección de Trabajo de clase, aplicación de GC ${ }^{\circledast}$. Tal como se muestra en la Figura garantizando la comunicación permanente, 14, se visualizan las clases diseñadas a lo largo de los actualización de las versiones y evidencias de cada una últimos años y cómo se acceden desde el perfil del de las jornadas de acompañamiento en la dirección de profesor:

trabajos de grado..

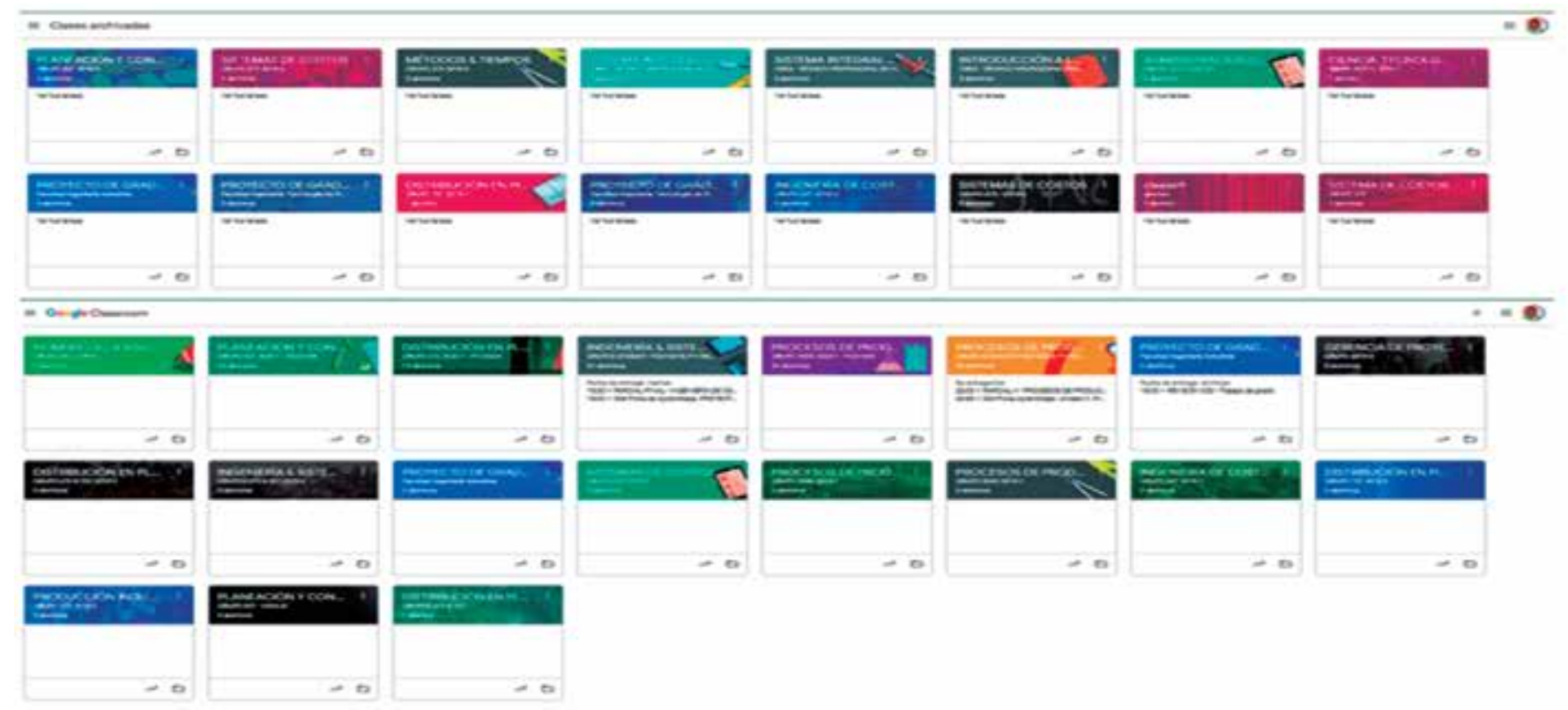

Fig. 14. Demostración de la visualización desde GC $C^{\oplus}$ de cursos diseñados entre 2018-2020(A) Fuente: Elaboración propia.

Recientemente se aplicó una encuesta en línea como muestra representativa de los estudiantes que a la fecha se encuentran cursando una clase desde $\mathrm{GC}^{\oplus}$, planteando cuatro preguntas cuyos resultados se aprecian en la Figura 15:

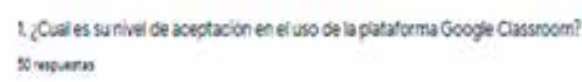

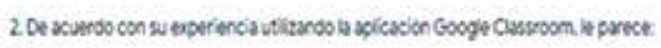
singusa
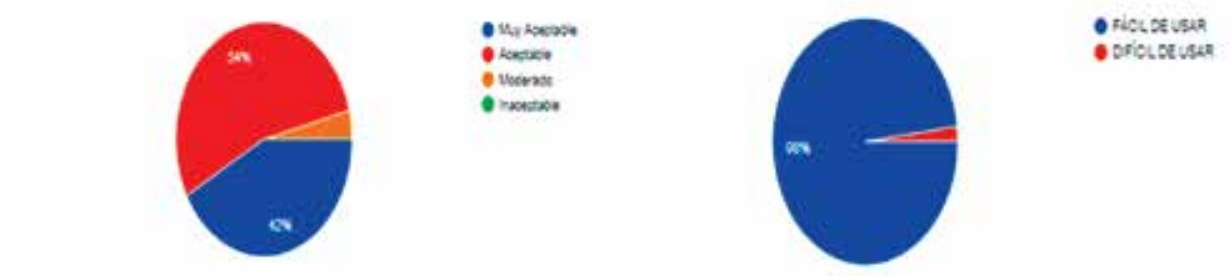

- richosusara - oriosectuga

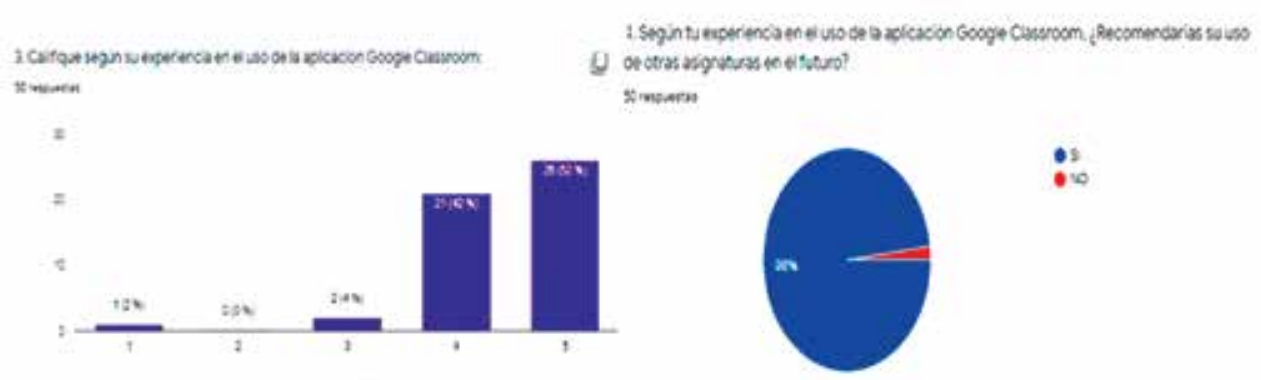

Fig. 15. Gráficos de resultados de la encuesta de satisfacción en el uso de GC ${ }^{\oplus}$. Fuente: elaboración propia - https://forms.gle/GC ${ }^{\oplus}$ mjpqBTmzanfV88 
Según los resultados que arroja la encuesta de satisfacción que se aplicó para la muestra de 51 estudiantes que están cursando clase en la aplicación $\mathrm{GC}^{\oplus}$, en alguna de las asignaturas, se concluye que:

$\rightarrow \quad ¿$ Cuál es su nivel de aceptación en el uso de la plataforma $\mathrm{GC}^{\circledR}$ ? El 96\% opinó que el nivel de aceptación del uso de las clases empleando $\mathrm{GC}^{\circledast}$ está entre aceptable y muy aceptable.

$\rightarrow$ De acuerdo con su experiencia utilizando la aplicación $\mathrm{GC}^{\circledR}$, ¿le parece? El $98 \%$ de los encuestados opinó que la experiencia en la utilización de la herramienta de las clases por medio de $\mathrm{GC}^{\circledast}$ es de fácil uso.

$\rightarrow$ Califique según su experiencia en el uso de la aplicación GC ${ }^{\circledast}$. Los encuestados puntuaron la experiencia en el manejo de la aplicación para el cumplimiento de sus compromisos académicos de la siguiente manera: con puntuación de 4 (42\%) y puntaje de 5 (52\%). Eso se interpreta como que el $94 \%$ de los estudiantes califican su experiencia con puntaje igual y superior de 4 .

$\rightarrow$ Según tu experiencia en el uso de la aplicación $\mathrm{GC}^{\circledR}$, ¿recomendarías su uso en otras asignaturas en el futuro? El 98\% de los estudiantes recomendaría el uso futuro de $\mathrm{GC}^{\oplus}$ para sus clases.

\section{CONCLUSIÓN}

Resultado de la amplia y gratificante experiencia presentada por más de dos años con el uso de la herramienta académica $\mathrm{GC}^{\circledast}$, el presente estudio de caso ha demostrado las cinco etapas y sus respectivos procedimientos básicos para la creación, estructuración y diseño de un curso, facilitando la gestión pedagógica de manera eficiente y eficaz. En este estudio investigativo se hace entrega de los formatos que servirán de guía en la presentación de los Acuerdos Pedagógicos, plan de clase, estructura de notas, referencias bibliográficas y planificación de compromisos académicos; para la planificación de Actividades de Aprendizaje por parte del profesor, se incluyen los formatos estandarizados para la entrega de compromisos académicos por parte de los estudiantes; asimismo, se hace demostración de los mecanismos de fácil uso y eficacia para la aplicación de talleres, tareas o evaluaciones con el empleo de formularios en línea, entre otros.

Esta investigación contribuirá en el desarrollo de nuevas alternativas pedagógicas orientadas para la realización de clases en modalidad presencial y aplicable también a distancia, favoreciendo la virtualización de los diferentes programas en las Instituciones de Educación Superior en Colombia, Latinoamérica y países hispanoparlantes, ampliamente adaptable a cualquier sistema educativo mundial.

\section{RECOMENDACIONES}

La evaluación e interpretación de los resultados obtenidos nos demuestran el alto nivel de aceptación que tiene la aplicación de esta herramienta académica en la gestión de curso por parte de los estudiantes. Es altamente recomendada para usos futuros en las diferentes asignaturas a cursar en los programas de Ingeniería y Tecnología de Producción Industrial de la Facultad de Ingenierías e, inclusive, a otros programas académicos de la institución.

Por los resultados presentados, queda en el proceso de verificación al interior de los Comités Académicos y de Planeación Institucional, en cabeza de la Vicerrectoría Académica, Coordinación Académica y facultades de los Programas Académicos de las IES, la socialización de este documento como caso experimental, brindando la oportunidad de vincular como alternativa pedagógica el uso ampliado de $\mathrm{GC}^{\circledast}$ e incluirlo como lineamiento institucional, como una opción estratégica para la gestión pedagógica de las clases de las asignaturas presenciales y su cobertura hacia las diferentes áreas del conocimiento de los demás 
programas académicos que oferta la Institución. El presente estudio de investigación servirá como punto de partida en el análisis y evaluación, en el manejo de las crisis externas que puedan afectar el normal desarrollo de los modelos presenciales, así como de insumo para la discusión y toma de decisiones en las mesas de trabajo pedagógico de las Instituciones de Educación Superior, examinando al interior de sus modelos las estrategias metodológicas del aprendizaje institucional y la efectividad de sus resultados en el proceso académico.

\section{REFERENCIAS BIBLIOGRÁFICAS}

Aja A. (2020, 30 de abril). Feliz día del niño en tiempos de Covid-19 " [en línea]. El Economista columna de opinión. https:// www.eleconomista.com.mx/opinion/FelizDia-del-Nino-en-tiempos-de-Covid-19-202 00430-0053.html

Al-Maroof, R. A. S., \& Al-Emran, M. (2018). Students acceptance of $\mathrm{GC}^{\oplus}$ : An exploratory study using PLS-SEM approach. International Journal of Emerging Technologies in Learning (iJET), 13(06), 112-123.

CESU. Colombia (2020, 4 de marzo de 2020). "El Ministerio de Educación, el Consejo Nacional de Educación Superior (CESU) y el Consejo Nacional de Acreditación (CNA) presentaron los lineamientos de actualización del Modelo de Acreditación en Alta Calidad para Instituciones $y$ Programas" [en línea]. Recuperado el 30 de abril de 2020 de, https://www.mineducacion. gov.co/1759/w3-article-393559.html?_nored irect $=1$

Real Academia Española. (2020). Definición de educación.

Kraus, G., Formichella, M.M. y Alderete, M.V (2019). El uso del GC ${ }^{\circledast}$ como complemento de la capacitación presencial a docentes de nivel primario. Revista Iberoamericana de Tecnología en Educación y Educación en Tecnología, 24, 79-90. DOI: 10.24215/18509959.24. e09

Henao, R. (2013). El concepto de crisis en el siglo XX-Una mirada general desde la contraposición existente alrededor de la teoría crítica y el positivismo lógico. Revista Movimentos Sociais e Dinâmicas Espaciais, Recife, 2, (2), 185-202.

González Mariño, J.C. (2008). TIC y la transformación de la práctica educativa en el contexto de las sociedades del conocimiento. RUSC. Revista Universidades y Sociedad del Conocimiento, 5 (2), 1-8. https://www.redalyc. org/articulo.oa?id=78011201003

Lavoz.com. (2020, 29 de abril). Por el coronavirus, el pico de actividad en Google fue cuatro veces mayor que el de la final de "Super Bowl", https:/www.lavoz.com.ar/tecnologia/porcoronavirus-pico-de-actividad-en-google-fue-cua tro-veces-mayor-que-de-final-de-super-

Colina, L. (2008). Las TIC en los procesos de enseñanza-aprendizaje en la educación a distancia. Revista de Educación, 28, 295-314.

Medaglia, O. (2015). Análisis de la incidencia de una estrategia pedagógica mediada por un web log en la asignatura de Ingeniería de métodos para el mejoramiento del trabajo autónomo en los estudiantes de Ingeniería Industrial de la Universidad de Pamplona (tesis de grado maestría). Universidad de Santander.

Ministerio de Educación Nacional-MEN. (2020). El Ministerio de Educación expide la Directiva 04 con orientaciones para las Instituciones de Educación Superior. https://www.mineducacion.gov.co/ portal/salaprensa/Noticias/394304:El-Ministeriode-Educacion-expide-la-Directiva-04-con-orienta ciones-para-las-Instituciones-de-Educacion-Supe rior 
Presidencia de Colombia (2020). Decreto 457 AUTOR

de 2020 mediante el cual se imparten instrucciones para el cumplimiento del Aislamiento Preventivo Obligatorio. https://www. mineducacion.gov.co/portal/salaprensa/Noticias/ 394357:Decreto-457-mediante-el-cual-se-impart en-instrucciones-para-el-cumplimiento-del-Aisla miento-Preventivo-Obligatorio

UNESCO (2015). Educación 2030: Hacia una educación inclusiva y equitativa de calidad y un aprendizaje a lo largo de la vida para todos.

Uribe Urán, A. P. (2013). Los factores que conforman el modelo de acreditación por alta calidad de programas académicos en Colombia, revisión desde el enfoque de la percepción. Desarrollo Gerencial, 5(2). http://revistas. unisimon.edu.co/index.php/desarrollogerencial/a rticle/view/489

Villacañas, J. (2013). Crisis: ensayo de definición. de Historia, 2, 121-140.

Oswaldo Medaglia Zapata: ingeniero industrial, especialista en administración de la informática educativa-UDES, magíster en gestión de la tecnología educativa - UDES. Experiencia docente por más de 12 años en Instituciones Universitarias privadas y públicas en programas de pregrado y postgrado. Actualmente docente de hora cátedra en la Institución de Universitaria Antonio José Camacho, para la Facultad de Ingenierías en los programas de Ingeniería Industrial y Tecnología de Producción Industrial; miembro del semillero de investigación-INTELIGO. Con experiencia de 20 años en empresas de manufactura y de servicios en gestión de la mejora en procesos productivos. Experto en gestionar y auditar sistemas de gestión integral HSEQ con base en las normas ISO, BASC y NTC. Auditor de Sistemas de Gestión Integral, aplicación de modelos de Lean Production, liderazgo en equipos de alto desempeño y participante en el equipo de implementación del MIPG Corporativo para empresa pública de Cali. Correo: omedaglia@profesores.uniajc.edu.co 